\title{
Unambiguous optical characterization of nanocolloidal gold films
}

\author{
Herbert Wormeester, ${ }^{*}$ E. Stefan Kooij, and Bene Poelsema \\ Solid State Physics, MESA + Research Institute, University of Twente, P.O. Box 217, 7500 AE Enschede, The Netherlands
}

(Received 15 April 2003; published 14 August 2003)

\begin{abstract}
The thin island film theory developed by Bedeaux and Vlieger [Optical Properties of Surface (Imperial College Press (2002)], is used to unambiguously analyze spectroscopic ellipsometry spectra of thin layers of nanocolloidal gold particles on silicon substrates covered by a natural oxide layer, for coverages up to $35 \%$. Due to the absence of isotropy in three dimensions, standard effective medium theories cannot be applied. Image dipole effects as well as the lateral distribution of colloids have to be taken into account as described by Bedeaux and Vlieger. Additonally we show that an effective image dipole has to be taken into account if colloidal particles are adsorbed on a substrate that is terminated with a thin $\mathrm{SiO}_{2}$ layer with a thickness that is smaller than the radius of the gold nanoparticles $(a=6.7 \mathrm{~nm})$. The natural oxide layer on our Si subtrates is only a fraction of this.
\end{abstract}

DOI: 10.1103/PhysRevB.68.085406

PACS number(s): 78.40.Kc, 78.67.Bf

\section{INTRODUCTION}

The properties of nanometer sized materials has increased the scientific interest in colloids of both the chemical and physics community significantly. The change of optical properties of metal colloids with decreasing size has been known for over a hundred years and has triggered many fundamental investigations. One of the applications of colloids involves the formation of a (diluted) single layer on a substrate. The optical analysis of such a heterogeneous thin film is not straightforward. ${ }^{1}$ It usually starts with the description of the thin film as an effective medium with theories established by Maxwell-Garnett ${ }^{2}$ and Bruggeman. ${ }^{3}$ However, both methods fail as the thin film cannot be described, even in a diluted state, as a three-dimensional (3D) isotropic medium. ${ }^{4}$ A proper description starts by treating the colloids as excess dipoles at the interface, which we will refer to as the bare dipole. As first described by Yamaguchi et al., ${ }^{5}$ the strength of this dipole is modified by an image dipole. Almost simultaneously the so-called "thin island film" theory for the low coverage limit was developed by Bedeaux and Vlieger, ${ }^{6}$ describing the optical response due to the influence of image dipoles in terms of dipole and quadrupole contributions to the bare dipole. The influence of lateral interaction between dipoles was analyzed by Haarmans et al. ${ }^{7,8}$ and Barrera et $a l^{9}{ }^{9}$ in the early 1990's. Especially the influence of densely packed square and hexagonal distribution of metal particles was considered. This work was used specifically for the analysis of deposited metal films, and recently also for hexagonal closed packed $5 \mathrm{~nm}$ Ag colloids. ${ }^{10}$ An extensive overview on the theory of the optical response of particles on a surface was given by Bedeaux and Vlieger. ${ }^{11}$

Much of the experimental work involving thin heterogeneous metal films deals with the high coverage regime. The deposition of colloids provides a simple route for the study of the optical response over a large coverage range, in which the optical properties of the single particles remain constant. In this article we analyze the optical response as measured with spectroscopic ellipsometry of a diluted layer of Au colloids (6.7 nm radius) on Si substrates. In previous work, we showed that for coverages between $20-30 \%$, a good corre- spondence between the surface density determined with atomic force microscopy (AFM) and the optically determined coverage is established. The optical response of the colloidal nanoparticles was treated in the bare dipole approximation. ${ }^{4}$ The good correspondence is in fact surprising since image dipole contributions are already relevant in the very low coverage regime and lateral interactions should play a role above $10 \%$ coverage. $^{7}$ In contrast, we found that below 20\% ellipsometry overestimated the coverage with this simple analysis. In this paper we will show that taking into account both image dipoles and lateral interaction up to quadrupole order solves this problem and a good comparison between calculated and measured spectra up to $40 \%$ surface coverage is obtained. For the lateral interaction, both an isotropic spatial distribution and an experimentally determined distribution are considered. Recently, we reported that for $\mathrm{Au}$ colloids in the coverage range considered, the lateral correlation function scales with coverage and exhibits a maximum at a specific distance, the preferred nearest neighbor distance. ${ }^{12}$ This was explained in terms of the electrostatic repulsion between the colloids which can be tuned by varying the ionic strength of the colloid suspension.

The strength of the image dipole is highly sensitive to the height $h$ of the center of the nanocrystal above an interface compared to its radius. The image dipole strength falls off with the third power of the ratio $a / h$. Evaluation of the influence of the image dipole is thus not straightforward. The 6.7 $\mathrm{nm}$ radius $\mathrm{Au}$ colloids are deposited on approximately 1.8 nm thick $\mathrm{SiO}_{2}$ layers on $\mathrm{Si}$ substrates. The thickness of the oxide layer is smaller than the nanocrystal radius and can no longer be viewed as the only medium which has to be considered in determining the contribution of the image dipole. On the other hand, taking only the silicon substrate into account is also not justified, since the extra $1.8 \mathrm{~nm}$ distance already reduces the image dipole strength by a factor 2 . An effective image dipole strength that considers the reflection and transmission of the electric field at both the ambient silicon oxide and the silicon oxide substrate interfaces will be introduced. We show that this effective image dipole strength is relevant for coverages above $10 \%$.

The colloid deposition and optical characterization by el- 
lipsometry was described previously. ${ }^{4}$ In the present work, low voltage high resolution scanning electron microscopy (SEM) is preferred over AFM for evaluating the number of nanoparticles per surface area. In the latter method, the convolution of tip and particle complicates the accurate determination of the number of particles per area, especially for higher coverages.

After a brief description of the thin island film theory, the effective image dipole strength is introduced and compared to the experimental results. An analysis of the difference between using an isotropic distribution and a distribution with a preferred lateral nearest neighbor distance is given.

\section{THIN ISLAND FILM THEORY}

The influence of image dipoles on the optical response of small particulates on a surface was considered by Yamaguchi et al. $^{5}$ and Bedeaux and Vlieger. ${ }^{6}$ The influence of the lateral distribution on the optical response was considered by Haarmans et al. ${ }^{7}$ In their treatment, they model the optical response of the particulates by an excess dipole at the interface. The magnitude of the dipole is given by the one particle polarizability along the interface and perpendicular to the interface $\gamma$ and $\beta$, respectively. Without image dipole and lateral interaction, the bare polarizabilities are defined as

$$
\gamma=\phi 4 a \varepsilon_{a} \frac{\varepsilon-\varepsilon_{a}}{\varepsilon+2 \varepsilon_{a}}
$$

$$
\beta=\frac{\phi 4 a}{\varepsilon_{a}} \frac{\varepsilon-\varepsilon_{a}}{\varepsilon+2 \varepsilon_{a}},
$$

where $\varepsilon$ and $\varepsilon_{a}$ are the dielectric functions of the particle and the ambient, respectively, and $\phi=n \pi a^{2}$ is the surface coverage with $a$ the particle radius and $n$ the area density. Note that an essential difference between the thin island film theory and, for instance, the description by Barrera ${ }^{9}$ is that (i) the thickness of the layer in the thin film theory is simply given through the influence of the particle radius $a$ on the strength of the polarizability and (ii) the anisotropic response due the finite thickness of the layer is embedded in the thin island film theory by the different reaction of the electromagnetic field, especially due to the presence of a surface on which the colloids are adsorbed, on the polarizabilities $\gamma$ and $\beta$. The optical properties of the colloids are embedded in the dielectric function of the noninteracting spherical particle. This dielectric function can, for instance, be experimentally obtained from an extinction measurement of the colloid solution. ${ }^{4}$

The incorporation of image dipole effects and lateral interaction with neighboring particles leads to a modification of these one particle polarizabilities. Haarmans and Bedeaux ${ }^{8,11}$ have shown that these changes are adequately described by an expansion of the potential distribution up to quadrupole order. The optical response is thus described as the response of an ensemble of noninteracting particles with effective polarizabilities $\gamma$ and $\beta$ given by

$$
\begin{aligned}
& \gamma_{q u}=\phi \frac{4}{3} a \varepsilon_{a} \frac{\left(\varepsilon-\varepsilon_{a}\right)\left[\varepsilon_{a}+L 1_{p}\left(\varepsilon-\varepsilon_{a}\right)\right]}{\left[\varepsilon_{a}+L_{p}\left(\varepsilon-\varepsilon_{a}\right)\right]\left[\varepsilon_{a}+L 1_{p}\left(\varepsilon-\varepsilon_{a}\right)\right]+\Lambda_{p}\left(\varepsilon-\varepsilon_{a}\right)^{2}} \\
& \beta_{q u}=\frac{\phi 4 a}{3 \varepsilon_{a}} \frac{\left(\varepsilon-\varepsilon_{a}\right)\left[\varepsilon_{a}+L 1_{z}\left(\varepsilon-\varepsilon_{a}\right)\right]}{\left[\varepsilon_{a}+L_{z}\left(\varepsilon-\varepsilon_{a}\right)\right]\left[\varepsilon_{a}+L 1_{z}\left(\varepsilon-\varepsilon_{a}\right)\right]+\Lambda_{z}\left(\varepsilon-\varepsilon_{a}\right)^{2}}
\end{aligned}
$$

Here, $L_{p}$ and $L_{z}$ represent the dipolar correction terms and $L 1_{p}$ and $L 1_{z}$, and $\Lambda_{p}$ and $\Lambda_{z}$ are the quadrupole depolarization factors. Using

$$
B_{s a}=\left(\varepsilon_{a}-\varepsilon_{s}\right) /\left(\varepsilon_{a}+\varepsilon_{s}\right)
$$

with $\varepsilon_{s}$ the dielectric function of the substrate these correction terms are given by

$$
\begin{aligned}
& L_{p}=\frac{1}{3}\left[1+2 \sqrt{\frac{\pi}{5}} I_{2}(\phi)+2 \sqrt{\frac{\pi}{5}} B_{s a} I_{2 r}(\phi)\right], \\
& L_{z}=\frac{1}{3}\left[1-4 \sqrt{\frac{\pi}{5}} I_{2}(\phi)+4 \sqrt{\frac{\pi}{5}} B_{s a} I_{2 r}(\phi)\right], \\
& L 1_{p}=\frac{2}{5}\left[1-\frac{8}{3} \sqrt{\pi} I_{4}(\phi)+\frac{8}{3} \sqrt{\pi} B_{s a} I_{4 r}(\phi)\right],
\end{aligned}
$$

$$
\begin{gathered}
L 1_{z}=\frac{2}{5}\left[1+4 \sqrt{\pi} I_{4}(\phi)+4 \sqrt{\pi} B_{s a} I_{4 r}(\phi)\right], \\
\Lambda_{p}=\frac{8}{35} \pi\left[I_{3}(\phi)^{2}-B_{s a}^{2} I_{3 r}(\phi)^{2}\right], \\
\Lambda_{z}=\frac{24}{35} \pi\left[I_{3}(\phi)^{2}-B_{s a}^{2} I_{3 r}(\phi)^{2}\right] .
\end{gathered}
$$

The integral quantities $I_{l}$ and $I_{l r}$, with $l=2,3,4$ take into account the spatial distribution of the nanoparticles on the surface, in terms of the correlation function $g(r)$. The influence of the particles is thus given by

$$
I_{l}=2 \phi \sqrt{\frac{2 l+1}{4 \pi}} \int_{2}^{\infty} \frac{g(r)}{r^{l}} P_{l}^{0}(0) d r .
$$


The integrand is normalized to the radius $a$. Since we are dealing with nonoverlapping particles, the minimum nearestneighbor distance is $2 a$. $P_{l}^{0}$ is the legendre polynomial, and as $P_{3}^{0}(0)=0$, this gives $I_{3}(\phi)=0$. The integral that describes the influence of the image dipoles is given by

$$
\begin{aligned}
I_{l r}= & \frac{(-1)^{l} \sqrt{\frac{2 l+1}{4 \pi}} P_{l}^{0}(1)}{2^{l+1}}+2 \phi(-1)^{l} \\
& \times \sqrt{\frac{2 l+1}{4 \pi}} \int_{2}^{\infty} \frac{r g(r)}{\left(r^{2}+4\right)^{(l+1) / 2}} P_{l}^{0}\left(\frac{2}{\sqrt{r^{2}+4}}\right) d r .
\end{aligned}
$$

\section{ISOTROPIC LATERAL DISTRIBUTION}

For an isotropic, i.e., a purely statistical distribution of nanocrystals $g(r)=1$ for $a>2$. The integrals $I_{l}$ and $\mathrm{I}_{l r}$ in Eqs. (6) and (7) can be evaluated analytically: ${ }^{8}$

$$
\begin{aligned}
I_{2}(\phi) & =-\sqrt{\frac{5}{4 \pi}} \frac{\phi}{2}, \\
I_{2 r}(\phi) & =\sqrt{\frac{5}{4 \pi}}\left(\frac{1}{8}-\frac{\phi}{4 \sqrt{2}}\right), \\
I_{3}(\phi) & =0, \\
I_{3 r}(\phi) & =\sqrt{\frac{7}{4 \pi}} \frac{1}{16}\left(-1+\frac{\phi}{\sqrt{2}}\right), \\
I_{4}(\phi) & =\frac{3}{64 \sqrt{\pi}} \phi, \\
I_{4 r}(\phi) & =\sqrt{\frac{9}{4 \pi}} \frac{1}{32}\left[1-\phi\left(\frac{1}{\sqrt{2}} \frac{3}{8}\right)\right] .
\end{aligned}
$$

In Fig. 1, $\gamma$ and $\beta$ are shown for both the bare dipoles and the modified values with up to quadrupole interactions taken into account. The dielectric function of the Au nanocolloidal particles was obtained as described previously. ${ }^{4}$ The strength of the image dipoles was evaluated by inserting the silicon dielectric function in Eq. (3). In the limit of zero coverage, due to the incorporation of the image dipole effect the slope of the curves are different from those obtained using the bare dipole approximation. This explains the previously reported deviation between the optically and AFM determined coverages in the low coverage regime. ${ }^{4}$ Above $20 \% \gamma$ shows an underestimation by the bare dipole model, while for $\beta$ an overestimation is noted. These two effects cancel each other for a coverage of about $20 \%$ and results in a good correspondence between the optical and AFM determined coverage. The image dipole effect is especially noteworthy since Si has a relatively high dielectric function compared to many oxide

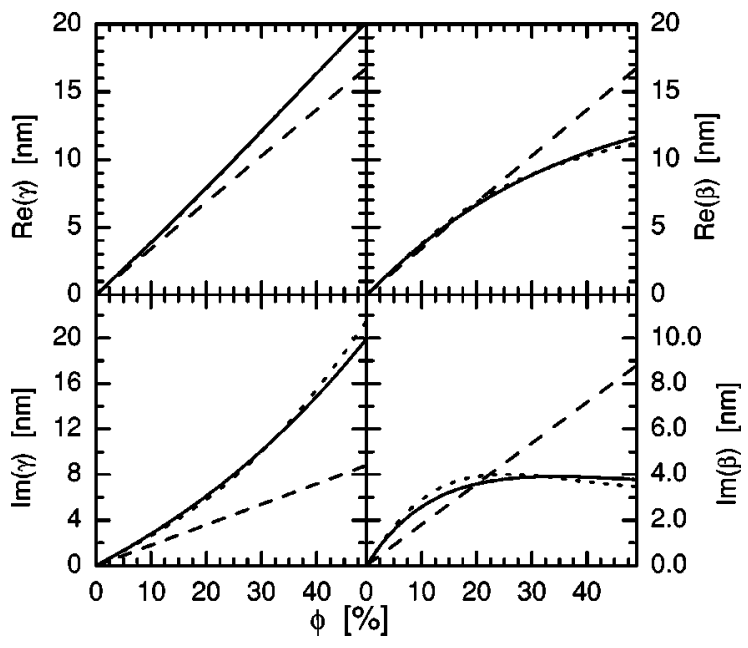

FIG. 1. Real and imaginary part of $\gamma$ and $\beta$ at $2.4 \mathrm{eV}(517 \mathrm{~nm})$ for the bare dipole approximation (dashed line) and after taking into account the influence of an isotropic distribution of colloids and their image dipole up to quadrupole order (solid line). Also shown are the values of the polarizabilities for a preferred distance distribution (dotted line).

materials commonly used as substrates. In this case $B_{\text {sa }}$ $=-0.89$ at a photon energy of $2.4 \mathrm{eV}$, i.e., a wavelength of $517 \mathrm{~nm}$, while, for instance, the value of $B_{s a}$ for quartz is almost 3 times smaller.

\section{THE IMAGE DIPOLE CONTRIBUTION}

The Au colloids with radius $a=6.7 \mathrm{~nm}$ were deposited on naturally oxidized silicon. The silicon oxide interface is covered with an approximately $0.3 \mathrm{~nm}$ thick layer of APTES, which gives a total layer thickness $d=1.8 \mathrm{~nm}$. As this layer is only a fraction of the particle radius $a$, the image dipole contribution has to be reconsidered. The strength of an image dipole contribution $B_{s a}$ for a dipole placed at distance $a+d$ above a homogeneous substrate falls of with $[a /(a+d)]^{3}$,

$$
B(d)=-\frac{\varepsilon_{s}-\varepsilon_{a}}{\varepsilon_{s}+\varepsilon_{a}}\left(\frac{a}{a+d}\right)^{3}=B_{s a}\left(\frac{a}{a+d}\right)^{3}
$$

with $\varepsilon_{s}$ and $\varepsilon_{a}$ the dielectric functions of the substrate and ambient, respectively. In the evaluation of the image dipole, usually the dielectric properties of the outermost layer on which colloids are deposited is used. However, this no longer holds when the thickness of the layer is equivalent or smaller than the radius of the colloid, such as in the present case. The nanoparticles have a radius three times larger than the thin layer on which they are deposited. The strength of the image dipole contribution is thus expected to be bound between values for the image dipole contribution given by a silicon substrate and that of a silicon oxide substrate, i.e., between $B_{s a}=-0.89$ and $B_{s a}=-0.365$, respectively. For image dipoles, the value $B_{s a}$ is treated as a reflection coefficient. ${ }^{13} \mathrm{An}$ expansion with reflection and transmission coefficients of both interfaces, combined with the attenuation factor given by the thickness of the layer $d$ yields an effective image dipole strength 


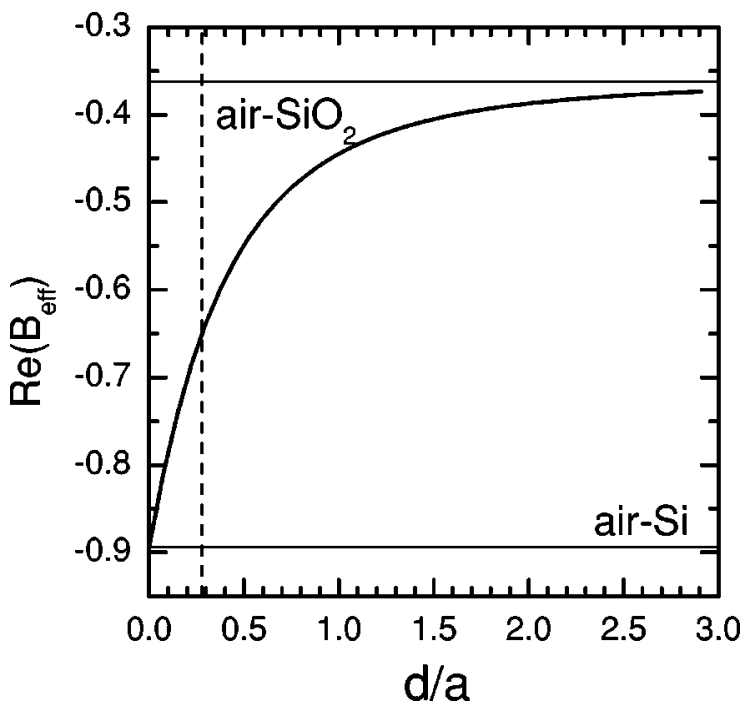

FIG. 2. The value of the real part of $B^{\text {eff }}$ at $2.4 \mathrm{eV}$ as a function of thickness $d$ of the $\mathrm{SiO}_{2}$ layer, normalized to the colloid radius $a$. The limit for $d=0$ is equal to the value for an air-Si interface and the limiting value for a large oxide thickness is equivalent to an air$\mathrm{SiO}_{2}$ interface. The dashed line indicates the value of $d / a$ for $d$ $=1.8 \mathrm{~nm}$.

$$
B^{\mathrm{eff}}(d)=\frac{B_{l a}+B_{s l}\left(\frac{a}{a+d}\right)^{3}}{1+B_{l a} B_{s l}\left(\frac{a}{a+d}\right)^{3}},
$$

where $B_{l a}$ and $B_{s l}$ represent the image dipole strengths for thinlayer-ambient and substrate-thinlayer interfaces. The value of $B^{\text {eff }}$ is plotted as a function of the $\mathrm{SiO}_{2}$ layer thickness in Fig. 2. The real part of $B^{\text {eff }}$ is considered only as the imaginary part is negligible at a photon energy of $2.4 \mathrm{eV}$. The rapid change of $B^{\text {eff }}$ with thickness leads to a considerably reduced value of the image dipole contribution already for thin oxide layers.

The influence of a thin silicon oxide layer on the polarizabilities $\gamma$ and $\beta$ is shown in Fig. 3. Especially for $\beta$ a marked difference is observed both in the real and imaginary parts. The most substantial difference is observed in the imaginary part of $\beta$ in the $10-30 \%$ coverage range.

Measured ellipsometric spectra for three different coverages are shown in Fig. 4. We analyzed the experimental ellipsometry spectra by fitting them to the thin island film theory. The optical response of the colloidal film in terms of the polarizabilities $\gamma$ and $\beta$ in Eqs. (2a) and (2b), respectively, is calculated using the Abeles matrix method. Details of this evaluation are described in Refs. 4,14. These references describe the matrix used in our calculation with the corresponding reflection coefficients. (Note that in this paper we follow the definition of the polarizabilities $\gamma$ and $\beta$ as also used in Ref. 11. In the two references for use of these polarizabilities in the Abeles formalism, a slightly different definition of the polarizabilities is used.) The surface coverage $\phi$ is the only free parameter in our fitting procedure. For all the models used in this work, i.e., a bare dipole, including

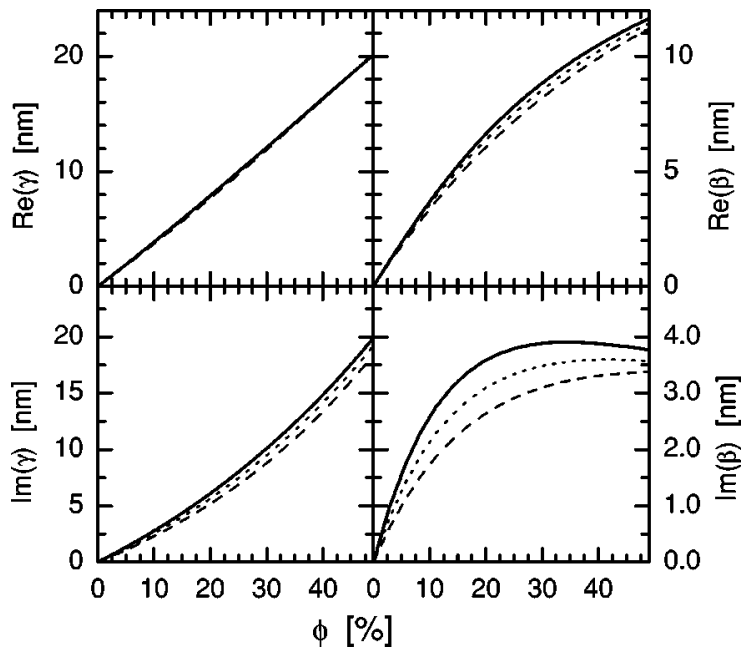

FIG. 3. The influence of different subtrates on the image dipole contribution for $\gamma$ and $\beta$. Depicted are the results obtained assuming a $\mathrm{Si}$ substrate (solid line), $\mathrm{SiO}_{2}$ (dashed line) and the effective substrate for a $d=1.8 \mathrm{~nm}$ oxide layer (dotted line), as described in the text.

various image dipole effects and lateral distributions, the quality of the fit is approximately equal. Especially the plasmon peak at $2.4 \mathrm{eV}$ is very sensitive not only for the coverage, but also for the size distribution and agglomeration of particles. The resonance energy shifts and simultaneously the peak broadens when two or more metal particles coagulate. ${ }^{15}$ We only consider the spectra in this analysis which did not show such broadening effects. A one to one correspondence between broadening and coagulation in the AFM and SEM pictures was found. The absence of such features in the presented spectra shows that we are able to deposit the citrate stabilized Au colloids up to a coverage of $35 \%$.

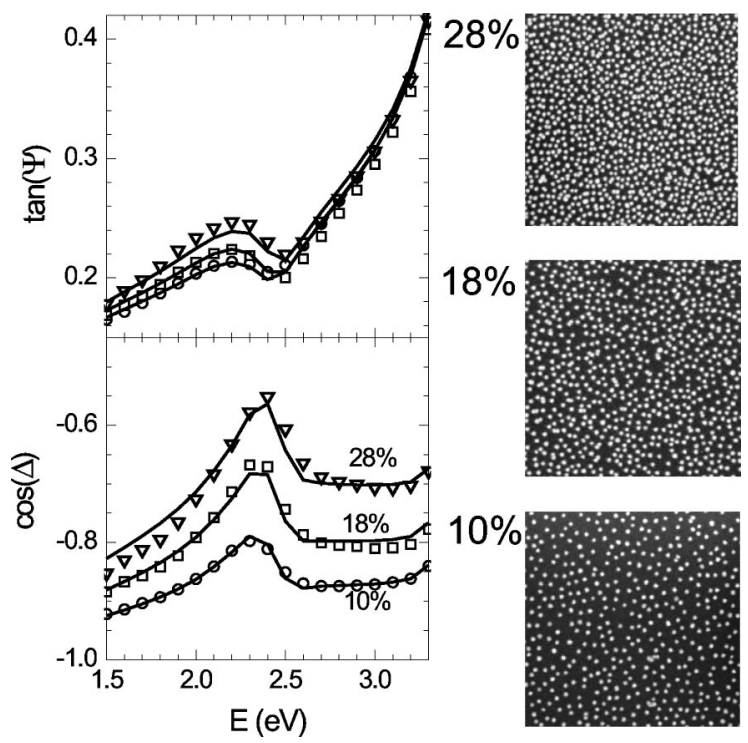

FIG. 4. Experimental ellipsometric spectra for different coverages of $\mathrm{Au}$ colloids on $\mathrm{SiO}_{2} / \mathrm{Si}$. The coverages as determined from the SEM images are indicated. The solid lines represent fit results using the thin island theory taking into account the effective image dipole and an isotropic lateral distribution of the nanocolloids. 


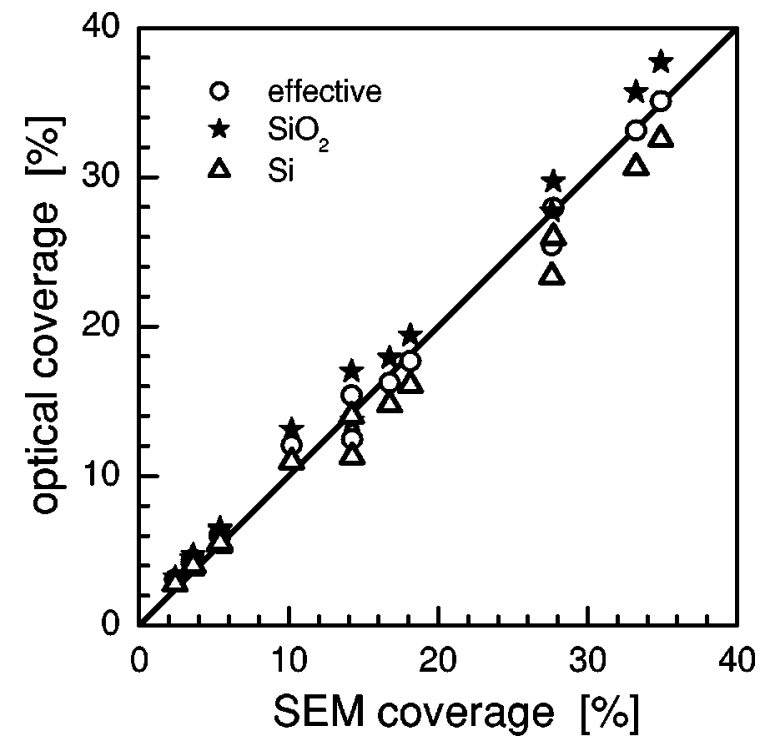

FIG. 5. Comparison of the coverage determined optically and with SEM. Three different models for the evalution of the image dipole contribution are depicted.

The influence of the image dipole contribution was evaluated in three different ways. Using the aforementioned fitting procedure, the optical coverage was determined from the spectra for both (i) a $\mathrm{Si}$ and (ii) a $\mathrm{SiO}_{2}$ substrate for the evaluation of the image dipole contribution. Also the optical coverage was determined with (iii) a $B^{\text {eff }}$, Eq. (10), for a layer with $d=1.8 \mathrm{~nm}$. Figure 5 shows the resulting optical coverages for the three aforementioned cases, as a function of the coverage determined using SEM. It is clear that if only $\mathrm{SiO}_{2}$ or $\mathrm{Si}$ is considered as a substrate in the calculation of the image dipole contribution, optically an overdetermination, respectively underdetermination results. The correction made by incorporating $B^{\text {eff }}$, yields a one to one correspondence between the optically and SEM determined coverages.

\section{PREFERRED DISTANCE LATERAL DISTRIBUTION}

Experimentally we found that the correlation functions which describe the lateral distribution of gold nanocrystals after deposition for various saturation coverages scale with respect to the nearest-neighbor distance $L$, see Ref. 12 . The scaled correlation function $g(r)$ is shown in Fig. 6. However, at coverages well below saturation, the distance distribution will not be this pronounced. Initially, i.e., far below the set saturation coverage, the colloids will be randomly distributed. In the series of experiments presented here, the coverage was not achieved through controlling the ionic strength, but by limiting the deposition time. Nevertheless, for coverages above $10 \%$, the scaled distribution function in Fig. 6 accurately represents the actual distribution. The coverage was found to scale in a coverage range of 10-35\% as

$$
\phi=\frac{\pi}{L^{2}},
$$

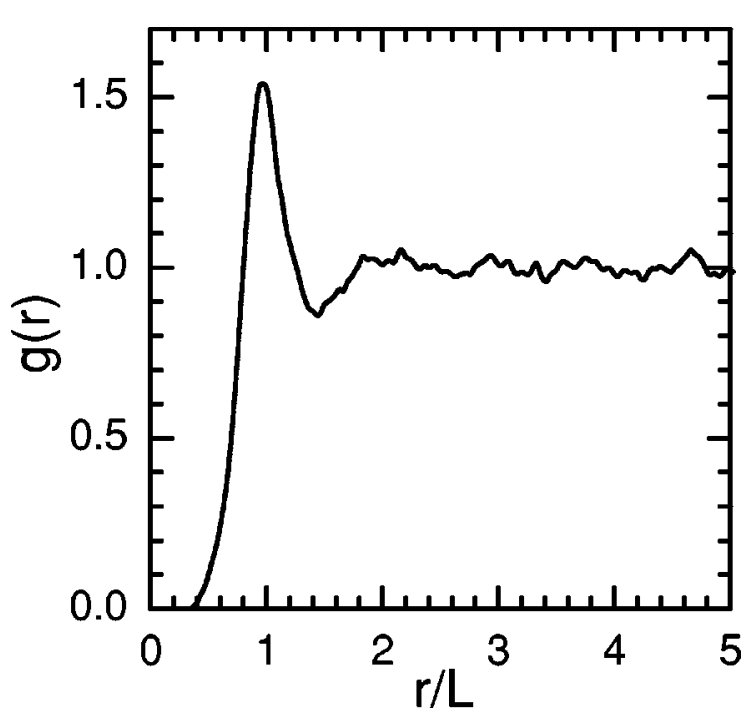

FIG. 6. Scaled correlation function of the spatial distribution of the Au colloids as determined with AFM (Ref. 12). The nearest neighbor distance is given by $L$.

i.e., the coverage of a square lattice with lattice parameter $L$, see Ref. 12.

With this correlation function, the integrals $I_{l}$ and $I_{l r}$ in Eqs. (6) and (7) related to the lateral distribution and the image dipole of this lateral distribution, respectively, can be evaluated, with the interparticle distance $L$ as parameter. Only for $I_{l}$, a substantial difference was found between the isotropic distribution and the preferred distance distribution. The difference only becomes significant for coverages above $5 \%$. The coverage dependent $I_{l}$ is used to calculate the par-

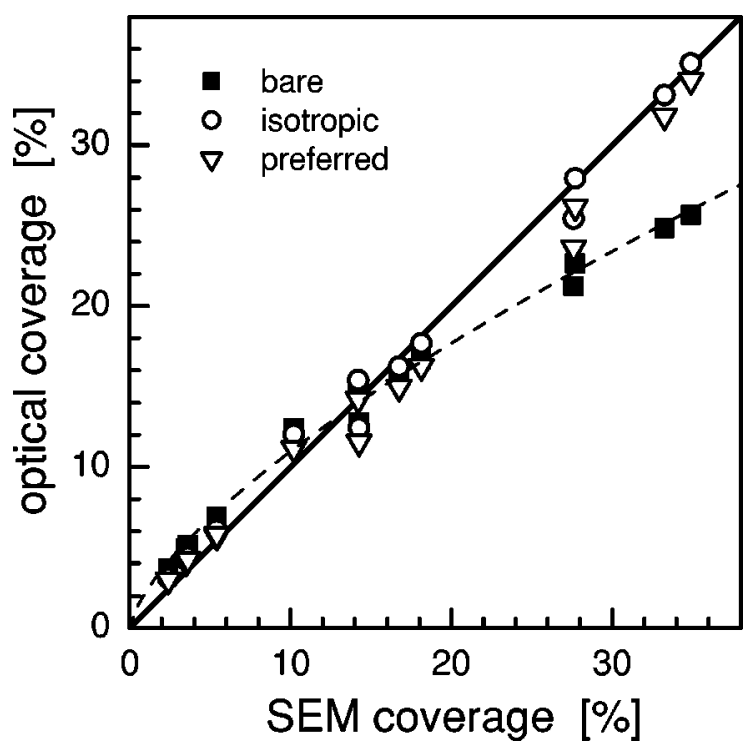

FIG. 7. The coverage as determined with spectroscopic ellipsometry, analyzed by taking into account the lateral distribution and image dipole effects as a function of the coverage determined with SEM. The lateral distribution was evaluated for an isotropic distribution (open circle) and the preferred lateral distribution (open triangle). Also the result for the bare dipole approximation is shown (full squares) with the dashed line as a guide to the eye. 
allel and perpendicular polarizabilities $\gamma$ and $\beta$, as depicted in Fig. 1.

The comparison of calculated values of $\gamma$ and $\beta$ for an isotropic distribution and the distribution over a square and hexagonal lattice, led Bedeaux et al. ${ }^{7,8,11}$ to the conclusion that below $40 \%$ hardly any difference between these distributions is noted optically. The optical coverage determined using only the bare polarizability [Eq. (1)], the isotropic distribution and for the preferred lateral distribution is shown in Fig. 7. The failure of using only the bare dipole is obvious from this graph in both the low coverage (overestimation below $15 \%$ ) and for the high coverage region (underestimation above 25\%). A significant difference between the isotropic distribution and the preferred lateral distribution is not observed. This supports the conclusion of Bedeaux et al. ${ }^{7,8,11}$ that below $40 \%$ the details of the lateral distribution is of no importance.

\section{CONCLUSION}

The ellipsometric spectra of Au nanocolloids deposited on $\mathrm{Si}$ substrates can be accurately described using the thin is-

land film theory developed by Bedeaux and Vlieger. ${ }^{11}$ The specific lateral distribution has little influence on the optical spectra for coverages up to $35 \%$. The image dipole contribution has a pronounced effect as naturally oxidized Si substrates are used. The thickness of this oxide layer is smaller than the colloid radius. This implies that a modified image dipole effect is required, the strength of which lies between that due to a bare $\mathrm{Si}$ substrate and an infinitely thick $\mathrm{SiO}_{2}$ layer. Only with this modified strength a one to one relation between the optically determined coverage and the coverage determined with SEM is obtained in the coverage range up to $35 \%$.

\section{ACKNOWLEDGMENTS}

We thank M. Smithers for performing the SEM measurements. We gratefully acknowledge a valuable discussion with D. Bedeaux. This work is part of the research program of the Stichting voor Fundamenteel Onderzoek der Materie (FOM), financially supported by the Nederlandse Organisatie voor Wetenschappelijk Onderzoek (NWO).

*Electronic address: h.wormeester@utwente.nl

${ }^{1}$ H. L. Zhang, S. D. Evans, and J. R. Henderson, Adv. Mater. (Weinheim, Ger.) 15, 531 (2003).

${ }^{2}$ J. C. Maxwell Garnett, Philos. Trans. R. Soc. London, Ser. A 203, 385 (1904).

${ }^{3}$ D. A. G. Bruggeman, Ann. Phys. (Leipzig) 24, 636 (1935).

${ }^{4}$ E. S. Kooij, H. Wormeester, E. A. M. Brouwer, E. van Vroonhoven, A. van Silfhout, and B. Poelsema, Langmuir 18, 4401 (2002).

${ }^{5}$ T. Yamaguchi, S. Yoshida, and A. Kinbara, Thin Solid Films 21, 173 (1973).

${ }^{6}$ D. Bedeaux and J. Vlieger, Physica A 67, 55 (1973).

${ }^{7}$ M. T. Haarmans and D. Bedeaux, Thin Solid Films 224, 117 (1993).

${ }^{8}$ M. T. Haarmans and D. Bedeaux, Thin Solid Films 285, 213
(1995).

${ }^{9}$ R. G. Barrera, M. Delcastillomussot, G. Monsivais, P. Villasenor, and W. L. Mochan, Phys. Rev. B 43, 13819 (1991).

${ }^{10}$ N. Pinna, M. Maillard, A. Courty, V. Russier, and M. P. Pileni, Phys. Rev. B 66, 045415 (2002).

${ }^{11}$ D. Bedeaux and J. Vlieger, Optical Properties of Surfaces (Imperial College Press, London, 2001).

${ }^{12}$ E. S. Kooij, E. A. M. Brouwer, H. Wormeester, and B. Poelsema, Langmuir 18, 7677 (2002).

${ }^{13}$ J. D. Jackson, Classical Electrodynamics (Wiley, New York, 1999).

${ }^{14}$ M. R. Bohmer, E. A. van der Zeeuw, and G. J. M. Koper, J. Colloid Interface Sci. 197, 242 (1998).

${ }^{15}$ U. Kreibig and M. Vollmer, Optical Properties of Metal Clusters (Springer, Berlin, 1995). 